\title{
Theoretical analysis of magnetic coupling in sandwich clusters $\mathbf{V}_{n}\left(\mathbf{C}_{6} \mathbf{H}_{6}\right)_{n+1}$
}

\author{
Hongming Weng, ${ }^{1, *}$ Taisuke Ozaki, ${ }^{1}$ and Kiyoyuki Terakura ${ }^{1,2,3}$ \\ ${ }^{1}$ Research Center for Integrated Science, \\ Japan Advanced Institute of Science and Technology, Nomi, Ishikawa 923-1292, Japan \\ ${ }^{2}$ Creative Research Initiative 'Sousei', \\ Hokkaido University, Sapporo 001-0021, Japan \\ ${ }^{3}$ Research Institute for Computational Sciences, \\ AIST, Tsukuba, Ibaraki 305-8568, Japan
}

(Dated: November 2, 2018)

\begin{abstract}
The mechanism of ferromagnetism stability in sandwich clusters $\mathrm{V}_{n}\left(\mathrm{C}_{6} \mathrm{H}_{6}\right)_{n+1}$ has been studied by first-principles calculation and model analysis. It is found that each of the three types of bonds between $\mathrm{V}$ and benzene $(\mathrm{Bz})$ plays different roles. $\mathrm{V} 3 \mathrm{~d}_{z^{2}}$ orbital, extending along the molecular axis, is weakly hybridized with Bz's HOMO-1 orbital to form the $\sigma$-bond. It is quite localized and singly occupied, which contributes $1 \mu_{B}$ to the magnetic moment but little to the magnetic coupling of neighboring $\mathrm{V}$ magnetic moments. The in-plane $\mathrm{d}_{x^{2}-y^{2}}, \mathrm{~d}_{x y}$ orbitals are hybridized with the LUMO of $\mathrm{Bz}$ and constitute the $\delta$-bond. This hybridization is medium and crucial to the magnetic coupling though the $\delta$ states have no net contribution to the total magnetic moment. $\mathrm{d}_{x z}, \mathrm{~d}_{y z}$ and $\mathrm{HOMO}$ of $\mathrm{Bz}$ form a quite strong $\pi$-bond to hold the molecular structure but they are inactive in magnetism because their energy levels are far away from the Fermi level. Based on the results of first-principles calculation, we point out that the ferromagnetism stability is closely related with the mechanism proposed by Kanamori and Terakura [J. Kanamori and K. Terakura, J. Phys. Soc. Jpn. 70, 1433 (2001)]. However, the presence of edge Bz's in the cluster introduces an important modification. A simple model is constructed to explain the essence of the physical picture.
\end{abstract}




\section{INTRODUCTION}

Since the discovery of ferrocene $\mathrm{Fe}\left(\mathrm{C}_{5} \mathrm{H}_{5}\right)_{2}$ and determination of its structure, intensive research of the sandwich complexes where a metal atom or ion is sandwiched with two organic molecules (mostly aromatic molecules) has been performed in the field of organometallic chemistry. ${ }^{1}$ The growing interests in these complexes are mainly due to their potential usages in catalysis, novel magnetic and optical materials and other various applications. Among these compounds, the 3d-transition-metal-organic-molecule complexes are particularly under current active study since molecular magnets are considered as potential candidates for future applications in high-density information storage. Therefore, since the discovery of ferromagnetism in vanadium-benzene $\mathrm{V}_{n} \mathrm{Bz}_{m}$ complexes, $, 2, \underline{3}$ many experimental and theoretical studies $4,5,6$ have been performed in order to shed light on the mechanism of their ferromagnetism stability. Now, it is generally accepted that for small size $\mathrm{V}_{n} \mathrm{Bz}_{m}$ complexes $(\mathrm{n} \leq 4)$, the energetically preferred structures are linear multiple-decker sandwich-like with $m=n-1, n$ and $n+1$. The Stern-Gerlach deflection experiments show that the magnetic moment in $\mathrm{V}_{n} \mathrm{Bz}_{n+1}$ increases linearly with $n \stackrel{3}{\underline{3}}$ Ab initio calculations have confirmed this $\frac{5,6}{6}$ though the magnitude of the measured magnetic moment is smaller than the theoretical value by about factor two, which is attributed to the spin relaxation effects during the measurement. $\stackrel{3}{2}$ Also, for the ideal infinite $(\mathrm{VBz})_{n=\infty}$ chain, first-principles calculations predicted that the ferromagnetic (FM) state is more stable than the anti-ferromagnetic (AFM) state. ${ }^{9,10}, 11$ A mechanism of double exchange interaction has been proposed $9,10,11$ to explain the ferromagnetism in this complex based on the electronic structures obtained with the density functional theory calculations within the local density approximation (LDA) or generalized gradient approximation (GGA).

However, it is well known that LDA or GGA does not reliably describe the localized state in transition metal compounds, especially for these low-dimensional complexes. Including on-site Coulomb interaction $U$ usually can improve the description and lead to a more realistic picture. In this paper, by performing $\mathrm{GGA}+U$ calculations, we find that the electronic structure is significantly modified. A mechanism, originally proposed by Kanamori and Terakura,,$\frac{12}{1}$ is found to be responsible for the stability of FM state both in infinite and finite V-Bz sandwich complexes. A similar mechanism was also proposed by Yabushita et al. $2,3,4$ for the ferromagnetism stability in $\mathrm{V}_{2} \mathrm{Bz}_{3}$. However, it is also found that the presence of 
edge $\mathrm{Bz}$ introduces an important modification for finite complexes. To show the essence of the physical picture, a simple model is constructed and the related parameters are fitted from the first-principles calculations of $n=2$ cases. The numerical model analysis is semiquantitatively consistent with the first-principles calculations for $n=2,3$ and 4 cases. The model calculation is also used to analyze the size dependence and configuration dependence of the energy cost for the magnetic moment reversal.

\section{METHODOLOGY}

All the first-principles calculations were performed using our software package OpenMX, 13 which is based on a linear combination of localized pseudo-atomic orbital (LCPAO) method. The PAOs are generated by a confinement potential scheme $\frac{14}{4}$ with a cutoff radius of 5.0 a.u. for hydrogen and carbon and 6.5 a.u. for vanadium, respectively. In the pseudopotential generation, the semicore $3 \mathrm{~s}$ and $3 \mathrm{p}$ states of $\mathrm{V}$ atom were included as valence states. The exchange correlation energy functional within GGA as parameterized by Perdew, Burke and Ernzerhof $(\mathrm{PBE})^{\underline{15}}$ is used. Double-valence and polarization orbitals were included and the basis set completeness was checked by optimizing the structure of single Bz molecule and body centered cubic V metal and comparing with the experimental values or all electron fullpotential calculations. The real-space grid technique ${ }^{16}$ was used with an energy cutoff of 250 Ry in numerical integrations and in the solution of the Poisson equation using the fast Fourier transformation algorithm. Hubbard $U$ is included by the approach proposed in Ref. 17 to improve the description of localized states like V 3d orbitals. All the geometrical structures of $\mathrm{V}_{n} \mathrm{Bz}_{n+1}$ complexes are fully optimized under each individual magnetic configuration until the forces are less than $1.0 \times 10^{-4}$ a.u.

\section{RESULTS AND DISCUSSIONS}

\section{A. Ideal infinite $(\mathrm{VBz})_{n=\infty}$ chain}

To understand the mechanism of ferromagnetism stability in V-Bz multiple-decker sandwich complex, we start by studying the bonding nature between $\mathrm{Bz}$ and $\mathrm{V}$. In general, the molecular axis (z-axis) is defined as the line that passes through the metal and the center of gravity of $\mathrm{Bz}$ molecule and classify the valence orbitals in terms of their pseudo-angular 
momenta around this axis. According to the symmetry, the six $\pi$-orbitals of benzene form one $p \sigma$ (HOMO-1), two $p \pi$ (HOMO), two $p \delta(\mathrm{LUMO})$ and one $p \phi(\mathrm{LUMO}+1)$ orbitals. When sandwiched by two Bz molecules, the five $3 \mathrm{~d}$ orbitals of the metal atom are classified to one $d \sigma\left(d_{z^{2}}\right)$, two $d \pi\left(d_{x z}\right.$ and $\left.d_{y z}\right)$, and two $d \delta\left(d_{x y}\right.$ and $\left.d_{x^{2}-y^{2}}\right)$ orbitals, and the $4 \mathrm{~s}$ orbital is classified to an $s \sigma$ orbital. $\stackrel{4}{10}$ To see the overall feature of the electronic structure, we performed first-principles calculations for both FM and AFM states of the ideal infinite $\mathrm{V}-\mathrm{Bz}$ chain within GGA. The total and partial densities of states (DOS) for the FM state are plotted in Fig. 1, which are basically the same as other calculations. $, \underline{9}, 10,11$ By examining these DOSs and the symmetry of orbital wave-functions, one can easily find: 1) The coupling between $p \sigma$ and $d \sigma$ is very weak, nearly non-interacting, making $d \sigma$ localized and largely spin-split. 2) The $p \pi$ and $d \pi$ orbitals have strong hybridization, making both bonding and antibonding states far away from the Fermi-level, either totally occupied or totally empty. They are responsible to the stability of the molecular structure but not active in magnetic or electronic properties. 3) The $p \delta-d \delta$ hybridization is of medium strength among the three bonds. $d \delta$ states are also spin split due to the Hund coupling with the spin polarized $d \sigma$ states. V atom has 5 electrons, which occupy the $d \sigma$ and doubly degenerate $d \delta$ bands. Therefore, the number of holes existing in the overlapping $d \sigma$ and $d \delta$ bands of the minority spin state is one. Due to this metallic feature, the double-exchange mechanism has been proposed to be the possible origin of ferromagnetism. ${ }^{9}$ 10,11 The FM state is more stable than the AFM state by $0.091 \mathrm{eV}$ per VBz (see Table I). The total magnetic moment is just $1 \mu_{B}$ per $\mathrm{VBz}$ unit with its integer value being consistent with the half-metallic feature of DOS. In fact, the local magnetic moment on $\mathrm{V}$ atoms is about $1.15 \mu_{B}$. The total magnetic moment is reduced to $1 \mu_{B}$ due to the negative magnetic moment on $\mathrm{Bz}$ being polarized through the $p \delta-d \delta$ hybridization. 10

However, for localized states like $d \sigma$ and $d \delta$, LDA or GGA is not generally reliable. GGA $+U$ could improve the description of the electronic structure. In the present calculation, $U$ is a parameter but we have confirmed that the qualitative features are not affected by changing $U$ from $2.0 \mathrm{eV}$ to $4.0 \mathrm{eV}$. As a typical case, the results with $U=3.0 \mathrm{eV}$ will be mostly shown below. The DOS of $\mathrm{GGA}+U$ calculation with $U=3.0 \mathrm{eV}$ is shown in Fig.2a for the FM state. As expected, the spin-splitting in $d \sigma$ and $d \delta$ is largely enhanced compared with that in GGA calculation. It is interesting to note that the broad band in the minorityspin state right below the Fermi level is mostly composed of the $p \delta$ states, which are LUMO 


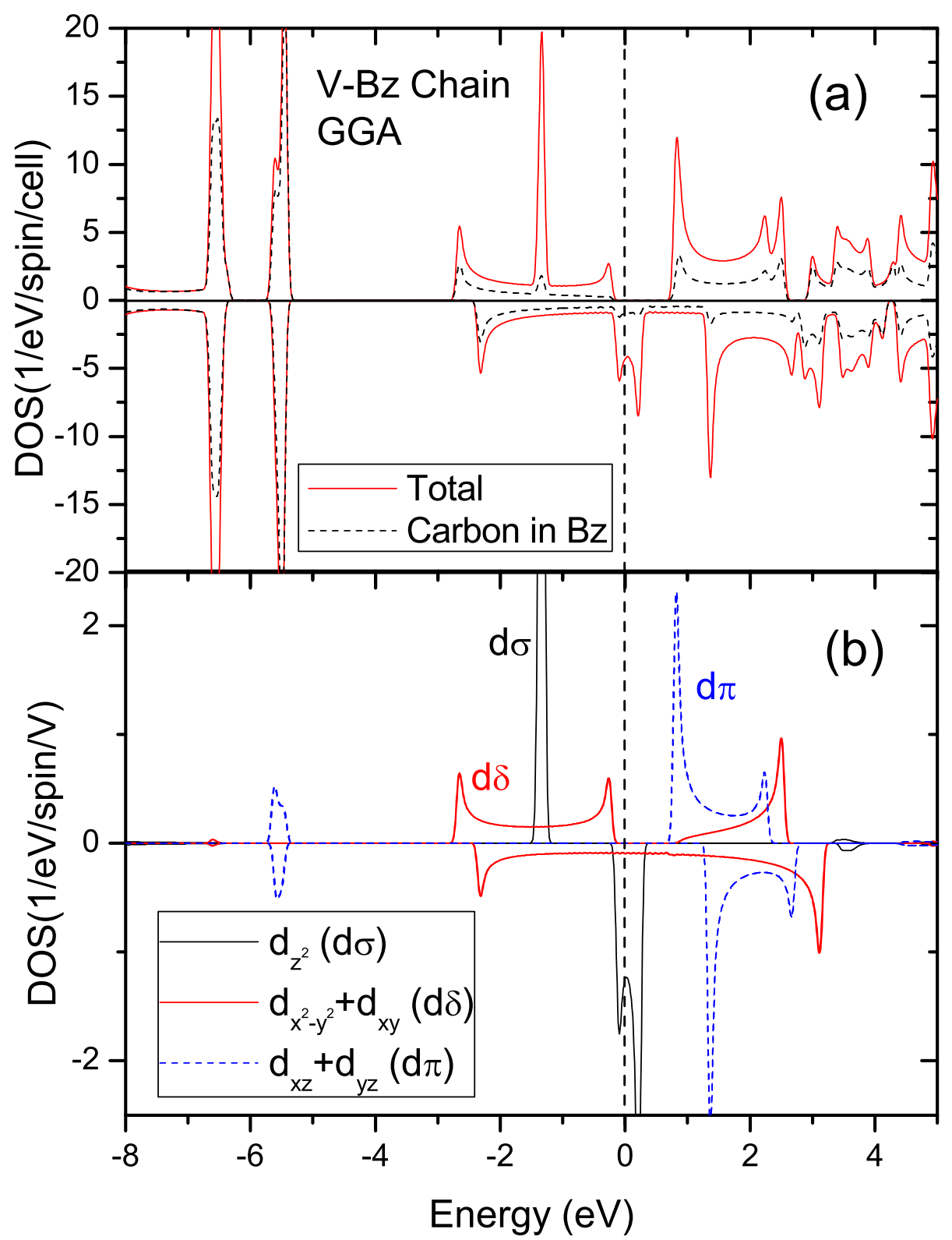

FIG. 1: (Color online) DOS of a FM infinite V-Bz chain calculated within GGA. (a) Total DOS and the partial DOS of carbons in Bz molecules; (b) partial DOS for each of V 3d orbitals.

in isolated Bz. Therefore,two $d \delta$ electrons of $\mathrm{V}$ are formally transferred to $p \delta$ states of Bz. The spin splitting in $d \delta$ states is now larger than the energy separation of $d \delta$ and $p \delta$ levels before the onset of the $p-d$ hybridization. (More detailed discussion will be given below.) A similar calculation for the AFM state gives the DOS shown in Fig.2b. Now, both the FM and AFM states are insulating and yet the stability of the FM state against the AFM state 

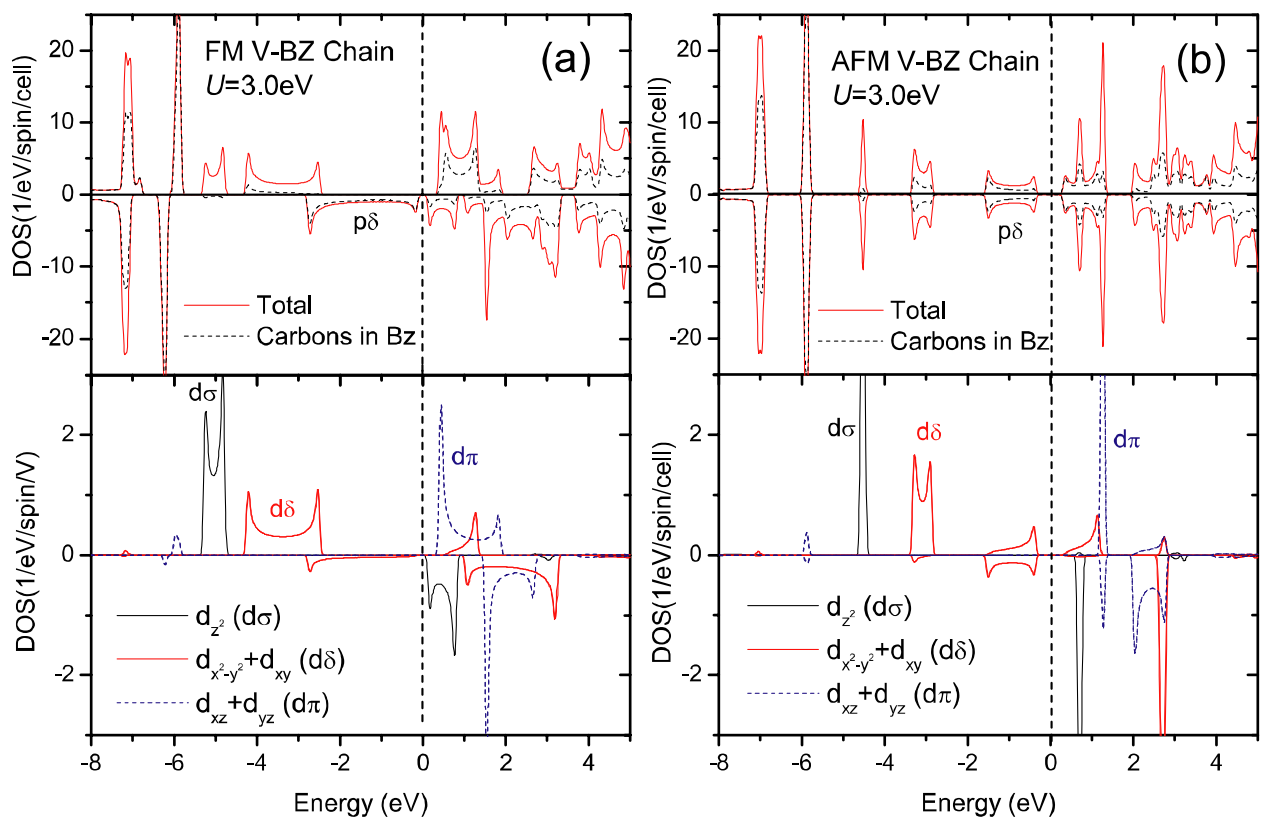

FIG. 2: (Color online) DOS of an infinite V-Bz chain calculated within GGA $+U(U=3.0 \mathrm{eV})$ for (a) FM and (b) AFM state. Upper panel of them is total and the partial DOS of carbons in Bz molecules, while the lower panel is partial DOS for each of V $3 \mathrm{~d}$ orbitals.

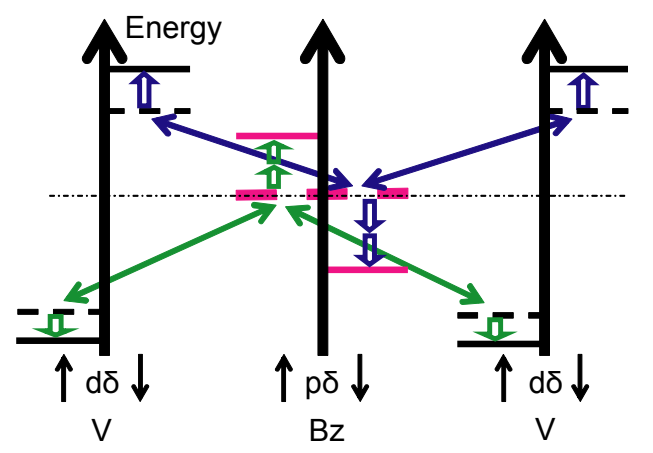

(a) FM

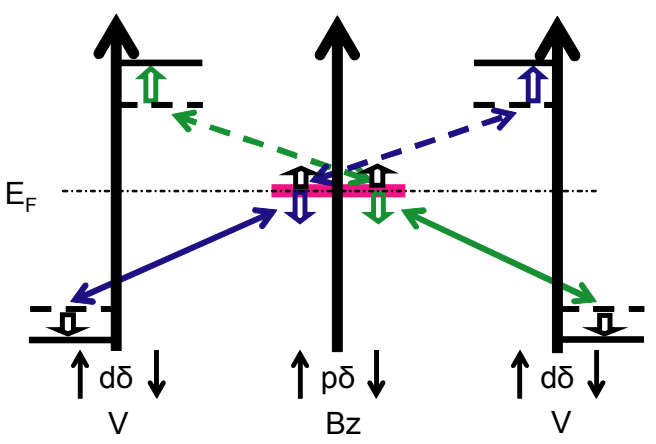

(b) AFM

FIG. 3: (Color online) A schematic picture describing the interactions between $\mathrm{V} d \delta$ and intervening $\mathrm{Bz} p \delta$ states in FM and AFM configurations. The thick vertical up arrow indicates the energy increasing direction and its left (right) side represents the majority- (minority-) spin channel. The horizontal solid (dashed) lines represent the energy levels of the corresponding state after (before) $p-d$ hybridization. The hollow arrows indicates the direction and magnitude of movements for each state. Fermi level is labeled by the horizontal dash-dotted line. 
is enhanced (see Table I). This implies that the mechanism of the ferromagnetism stability is not the double exchange. In the following, we further analyze the electronic structure in detail and elucidate the mechanism of the magnetic coupling between $\mathrm{V}$ magnetic moments.

As $d \sigma-p \sigma$ hybridization is weak, $d \sigma$ states does not make significant contribution to the magnetic coupling. Moreover, even if there is any contribution from them it will be AFM superexchange. The magnetic coupling is mostly governed by the $d \delta$ and $p \delta$ states. Therefore, we will focus only on the $\delta$ states. Figure 3 is a schematic diagram showing the basic feature of $p-d$ hybridization in the $\delta$ states. Due to the strong exchange splitting of $d \delta$ states, their majority-spin states are below the $p \delta$ states and fully occupied. On the other hand, the minority-spin $d \delta$ states are above the $p \delta$ states and empty. In the FM configuration of the $\mathrm{V}$ magnetic moments, the majority-spin $p \delta$ states are strongly pushed up in energy by the $p-d$ hybridization and vice versa in the minority-spin state. In the AFM configuration, on the other hand, the energy shift of $p \delta$ states is much reduced because the hybridization with the two neighboring $d \delta$ states produces energy shifts with opposite sign. In addition to this, the energy shift is the same in both spin states. One additional comment is that the apparent exchange splitting of $d \delta$ states is enhanced by the $p-d$ hybridization in both the FM and AFM states. Based on the observation of the energy shift scheme, we show a schematic DOS for $\delta$ states in the FM state in Fig. 4. In the absence of $p-d$ hybridization, the doubly degenerate $p \delta$ bands are half filled (Fig.4a). With the $p-d$ hybridization which produces large energy shifts in the $p \delta$ states (Fig.3a), the DOS is modified to the one shown in Fig.4b, which captures the essential feature of the $\delta$ symmetry part of the DOS in Fig.2a. Now, the electrons originally occupying the majority-spin $p \delta$ bands in Fig. 4 a are transferred to the minority-spin $p \delta$ bands as shown in Fig.4b, which produces a large energy gain in the FM state. A large negative spin polarization on $\mathrm{Bz}$ is also naturally explained. This is exactly the same story proposed by Kanamori and Terakura. $\frac{12}{2}$ The importance of the spin polarization in the intervening $\mathrm{Bz}$ for the ferromagnetism stability was also pointed out by Yabushita et al. for $\mathrm{V}_{2} \mathrm{Bz}_{3}$ cluster, 2,3,4

As shown in Table I, the total energy difference between FM and AFM states increases as $U$ value increases. Although the local magnetic moment on $\mathrm{V}$ ion increases with $U$, the negative polarization on $\mathrm{Bz}$ also increases and keeps the total magnetic moment per $\mathrm{VBz}$ unit the same as $1.0 \mu_{B}$, which is produced by $d \sigma$ states. 
TABLE I: Magnetic moment on $\mathrm{V}\left(\mu_{B}\right)$ and total energy difference between FM and AFM states $\left(\Delta \mathrm{E}=\mathrm{E}_{F M}-\mathrm{E}_{A F M}\right)$ calculated with different $U$ values.

\begin{tabular}{|c|c|c|c|c|}
\hline \multirow{2}{*}{$\mathrm{U}(\mathrm{eV})$} & \multicolumn{2}{|c|}{ Infinite V-Bz Chain } & \multicolumn{2}{c|}{$\mathrm{V}_{2} \mathrm{Bz}_{3}$} \\
\cline { 2 - 5 } & Magnetic moment on $\mathrm{V}$ & $\Delta \mathrm{E} / \mathrm{V}(\mathrm{eV})$ & Magnetic moment on V & $\Delta \mathrm{E} /$ cluster $(\mathrm{eV})$ \\
\hline 0.0 & 1.15 & -0.091 & 1.10 & -0.003 \\
\hline 1.0 & 1.35 & -0.113 & 1.17 & -0.004 \\
\hline 2.0 & 1.67 & -0.109 & 1.29 & -0.004 \\
\hline 3.0 & 2.03 & -0.184 & 1.50 & -0.005 \\
\hline 4.0 & 2.23 & -0.251 & 1.82 & -0.007 \\
\hline
\end{tabular}

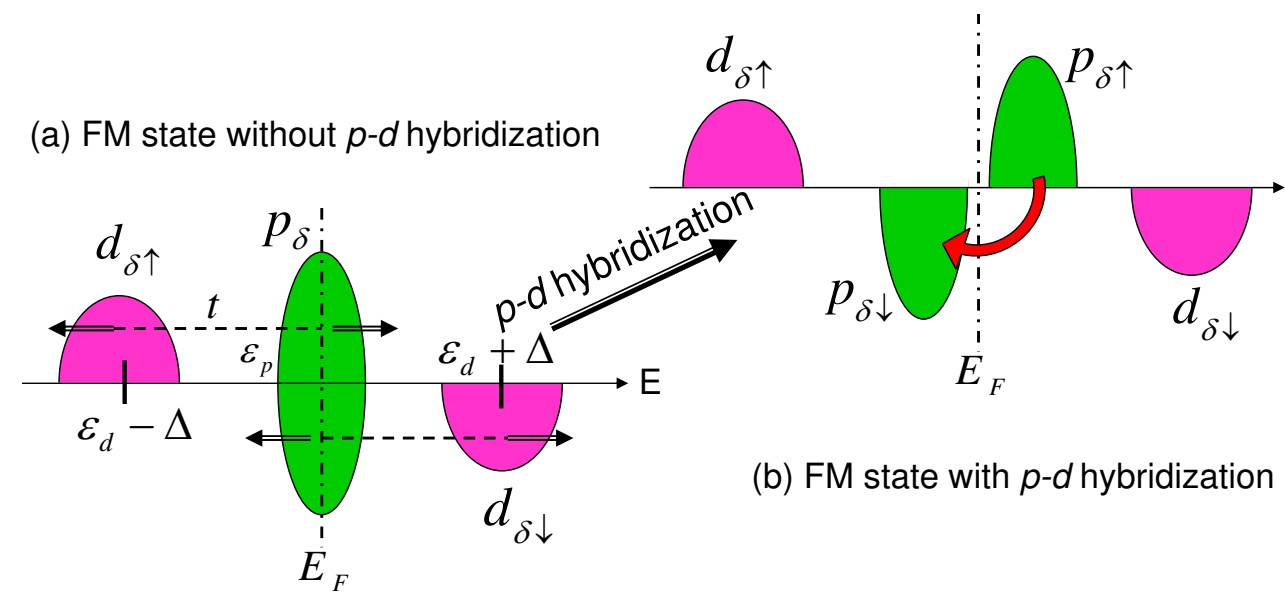

FIG. 4: (Color online) Schematic pictures describe the mechanism in which the $p-d$ hybridization stabilize the FM states. The DOS (a) without and (b) with $p-d$ hybridization is plotted. The energy gain due to the charge transfer is indicated.

\section{B. Finite $\mathbf{V}_{n} \mathbf{B} \mathbf{z}_{n+1}$ clusters}

Now, we come to the study of finite size sandwich clusters $\mathrm{V}_{n} \mathrm{Bz}_{n+1}$. For $n=1$, the spinpolarized $\mathrm{VBz}_{2}$ with $\mathrm{S}=1 / 2$ has lower energy than the non-magnetic one, which is consistent with the experimental measurement. For $n=2$, as shown in Table I the negative total energy difference between FM and AFM states indicates the FM state is more stable, being also consistent with experimental observations. However, the value of the energy difference is only several meV, much smaller than that in the infinite chain. Other calculations also give the energy difference less than $10 \mathrm{meV} \stackrel{3,5}{\underline{5}}$ Before presenting further detailed analysis, the 
following comment on Table I may be needed for the comparison of energies. The total energy difference is for one $\mathrm{VBz}$ unit in the infinite chain, while it is for $\mathrm{V}_{2} \mathrm{Bz}_{3}$ in the cluster. A justification of this seemingly strange comparison comes from an expectation that the magnetic coupling comes mostly from the energy change associated with the spin polarization of the intervening $\mathrm{Bz}$ between the $\mathrm{V}$ atoms. In this sense, both units have only one $\mathrm{Bz}$ shared by two neighboring $\mathrm{V}$ atoms.

According to the physical picture described in the infinite chain, the magnetic coupling in $\mathrm{V}_{2} \mathrm{Bz}_{3}$ can be studied by a simple tight-binding model which takes account of the hybridization between $p \delta$ and $d \delta$ states. For example the model Hamiltonian matrix for the AFM state is given as

$$
\left|\begin{array}{ccccc}
\varepsilon_{p} & t^{\prime} & 0 & 0 & 0 \\
t^{\prime} & \varepsilon_{d}-\Delta & t & 0 & 0 \\
0 & t & \varepsilon_{p} & t & 0 \\
0 & 0 & t & \varepsilon_{d}+\Delta & t^{\prime} \\
0 & 0 & 0 & t^{\prime} & \varepsilon_{p}
\end{array}\right|
$$

where $\epsilon_{p}$ and $\epsilon_{d}$ are the on-site energies of $p \delta$ and $d \delta$ levels, respectively, and $2 \Delta$ is the exchange splitting. In order to see the role of the edge Bz explicitly, the hopping integral between $d \delta$ and $p \delta$ is expressed as $t^{\prime}$ for the edge Bz part and $t$ for the middle Bz part.

To remove the edge Bz's, we adopt a cyclic boundary condition in the $(\mathrm{VBz})_{2}$ cluster. The Hamiltonian, which is identical to the $\Gamma$ point expression of an infinite chain, is written as

$$
\left|\begin{array}{cccc}
\varepsilon_{p} & t & 0 & t \\
t & \varepsilon_{d}-\Delta & t & 0 \\
0 & t & \varepsilon_{p} & t \\
t & 0 & t & \varepsilon_{d}+\Delta
\end{array}\right|
$$

The eigenvalues of these Hamiltonians can be calculated easily. Assuming $\epsilon_{p}=\epsilon_{d}=\epsilon_{0}$ and taking the approximation that $|t / \Delta|<<1.0$, we show the energy diagrams in Fig. 5. In the $n=2$ case, there are 10 electrons introduced by two $\mathrm{V}$ atoms. Two of them occupy the $d \sigma$ states and the other 8 electrons should occupy the $d \delta$ and $p \delta$ states depicted here. Note that both $d \delta$ and $p \delta$ states have double orbital degeneracy. Fermi level can be determined as shown in the energy diagrams. In order to understand the overall features of energetics among four different configurations shown in Fig.5, we assume $t^{\prime}=t$, which is reasonable 
since the $\mathrm{V}$-Bz distance is almost equally spaced. Then we note the following aspects in the energy diagram.

1) As for the energy levels originating from $d \delta$ states, the sum of the occupied part of orbital energies is the same for all cases shown in Fig.5. Note that the spin degeneracy has to be taken into account in the case of AFM state. Therefore, the total energy difference among the four cases shown in Fig.5 comes entirely from the energy diagram associated with $p \delta$ states.

2) In the case of FM state, even for the energy levels originating from $p \delta$ states, the sum of occupied part of orbital energies is the same for both isolated cluster and cyclic boundary condition.

3) In the case of AFM state with cyclic boundary condition, as there is no shift of energy levels of $p \delta$ states, the FM state is definitely lower in energy by $8 t^{2} / \Delta$ per cluster.

4) In the case of AFM state for isolated cluster, the energy of one of the $p \delta$ states is lowered due to the spin polarization at the edge Bz. Therefore the edge Bz contributes to the stabilization of AFM state of the isolated cluster. The FM state is still lower in energy than the AFM state but now only by $4(2-\sqrt{3}) t^{2} / \Delta$ per cluster. This is basically the reason for the significant reduction in the stability energy of the FM state against the AFM state in the isolated cluster as shown in Table I.

Now we give some additional comments on the role of edge $\mathrm{Bz}$ in the stabilization of AFM state in isolated cluster. If there is no electronic coupling among the Bz's, the energy level shift of the edge $p \delta$ state will be simply $-t^{\prime 2} / \Delta$. Then the stability energy of the FM state against the AFM state for the isolated cluster will become $4 t^{2} / \Delta$, just half of that for the cyclic boundary condition, as is naively expected. However, because the $p \delta$ states of the three Bz's form a coupled degenerate system, all the three $p \delta$ states contribute to the polarization of the edge $\mathrm{Bz}$ and the actual energy shift is much larger as shown in Fig.5. This can be seen in the AFM DOS of Fig. 6. The state around $-1.0 \mathrm{eV}$ is the magnetically polarized $\mathrm{Bz} p \delta$ state which consists mainly of the edge $\mathrm{Bz}$ state and also partly of the middle Bz state. Although the FM state is still more stable than the AFM state for any values of $t^{\prime}$ and $t$, the energy difference is reduced to $2\left(t / t^{\prime}\right)^{2}\left(t^{2} / \Delta\right)$ when $\left|t / t^{\prime}\right|<<1.0$.

In order to make the model analysis more quantitative, the parameters in the model are adjusted to reproduce the energy diagram obtained by the first-principles calculation. The total and partial DOS for the AFM and $\mathrm{FM} \mathrm{V} \mathrm{V}_{2} \mathrm{Bz}_{3}$ calculated with $U=3.0 \mathrm{eV}$ are plotted 


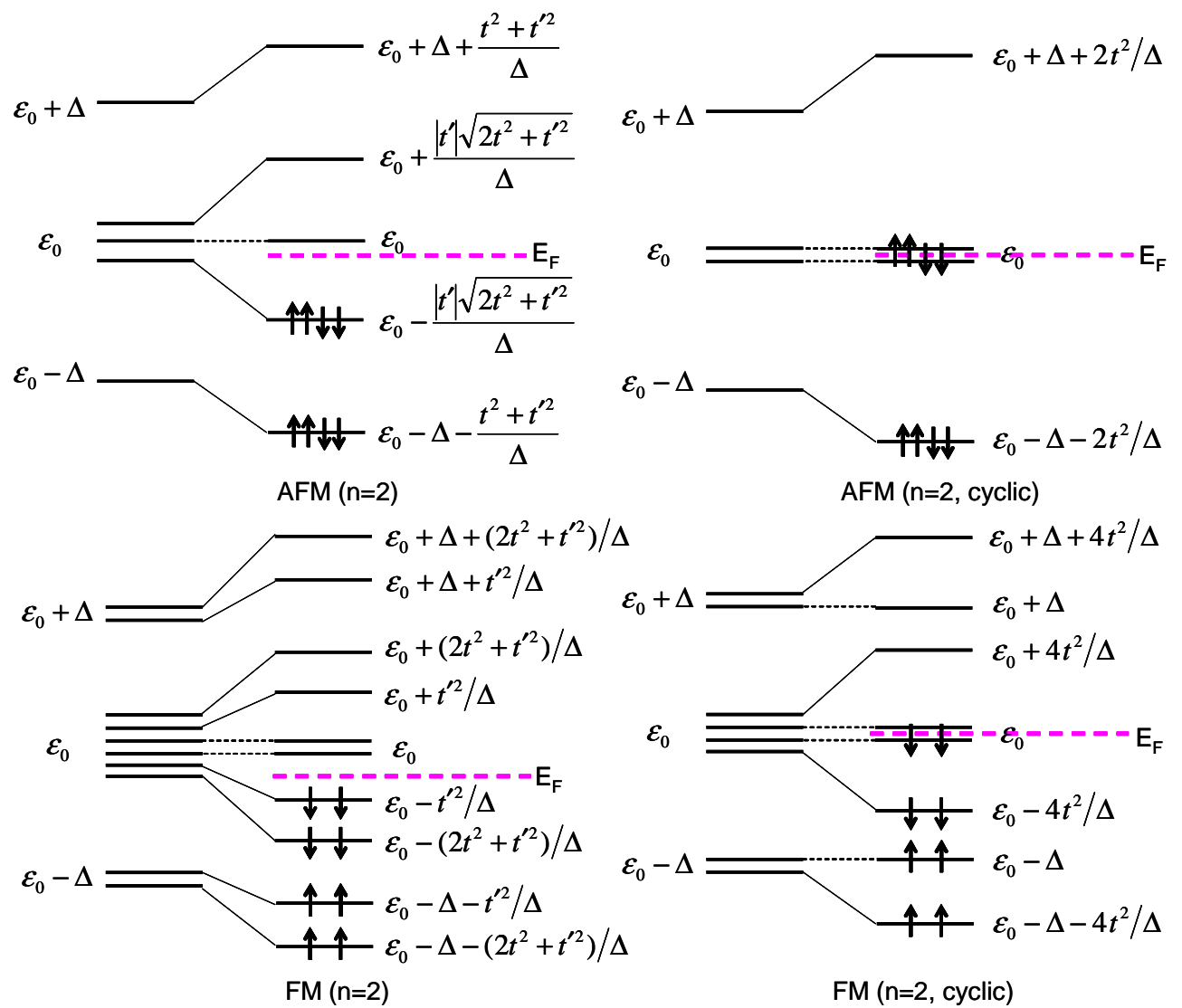

FIG. 5: (Color online) Energy diagrams for AFM and FM V $\mathrm{Bz}_{3}$ cluster and (VBz) 2 with cyclic boundary condition. $\epsilon_{p}=\epsilon_{d}=\epsilon_{0}$ is assumed and the energy levels are given only in the lowest order of $t^{2} / \Delta$ and $t^{\prime 2} / \Delta$.

TABLE II: Magnetic configuration (Arrow indicates the direction of local moment on V atoms and vertical lines represent Bz's) of $\mathrm{V}_{3} \mathrm{Bz}_{4}$, number of Bz's sandwiched with antiparallel $\mathrm{V}$ magnetic moments (represented by vertical dotted lines) and the energy difference (in $\mathrm{eV}$ ) relative to the FM state from model calculation. Those in parentheses are from first-principles GGA $+U$ calculations with $U=3.0 \mathrm{eV}$. The structure is optimized for each magnetic configuration in the GGA $+U$ calculations, while $t=t^{\prime}=1.648 \mathrm{eV}$ is used in the model analysis.

\begin{tabular}{|c|c|c|}
\hline magnetic configuration & No. of $\uparrow \vdots \downarrow$ pairs & Energy difference relative to FM state \\
\hline$|\uparrow| \uparrow|\uparrow|$ & 0 & 0.0 \\
\hline$|\uparrow| \uparrow \vdots \downarrow \mid$ & 1 & $0.033(0.018)$ \\
\hline$|\uparrow \vdots \downarrow \vdots \uparrow|$ & 2 & $0.060(0.037)$ \\
\hline
\end{tabular}



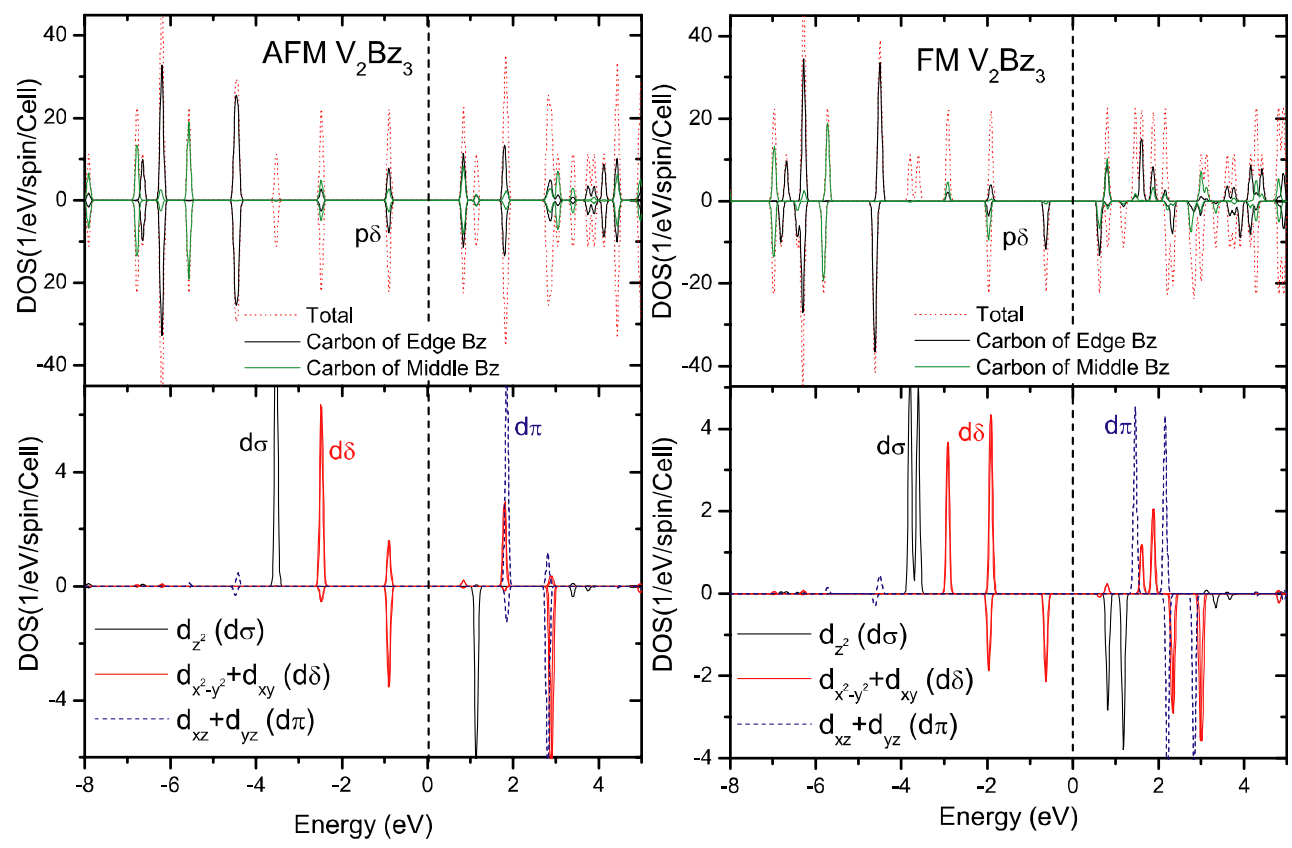

FIG. 6: (Color online) Total and partial DOS for AFM and FM V ${ }_{2} \mathrm{Bz}_{3}$ cluster calculated with $\mathrm{GGA}+U$ and $U=3.0 \mathrm{eV}$.

TABLE III: Magnetic configurations (Arrow indicates the direction of local moment on V atoms and vertical lines represent Bz's) of $\mathrm{V}_{4} \mathrm{Bz}_{5}$, number of Bz's sandwiched with antiparallel V magnetic moments (represented by vertical dotted lines) and the energy difference (in eV) relative to the FM state from model calculation. Those in parentheses are from first-principles GGA $+U$ calculations with $U=3.0 \mathrm{eV}$. The structure is optimized for each magnetic configuration in the GGA $+U$ calculations, while $t=t^{\prime}=1.648 \mathrm{eV}$ is used in the model analysis.

\begin{tabular}{|c|c|c|}
\hline magnetic configuration & No. of $\uparrow \vdots \downarrow$ pairs & Energy difference relative to FM state \\
\hline$|\uparrow| \uparrow|\uparrow| \uparrow \mid$ & 0 & 0.0 \\
\hline$|\uparrow| \uparrow|\uparrow \vdots \downarrow|$ & 1 & $0.037(0.036)$ \\
\hline$|\uparrow| \uparrow \vdots \downarrow|\downarrow|$ & 1 & $0.051(0.056)$ \\
\hline$|\uparrow \vdots \downarrow| \downarrow \vdots \uparrow \mid$ & 2 & $0.073(0.065)$ \\
\hline$|\uparrow| \uparrow \vdots \downarrow \vdots \uparrow \mid$ & 2 & $0.077(0.082)$ \\
\hline$|\uparrow \vdots \downarrow \vdots \uparrow \vdots \downarrow|$ & 3 & $0.105(0.103)$ \\
\hline
\end{tabular}




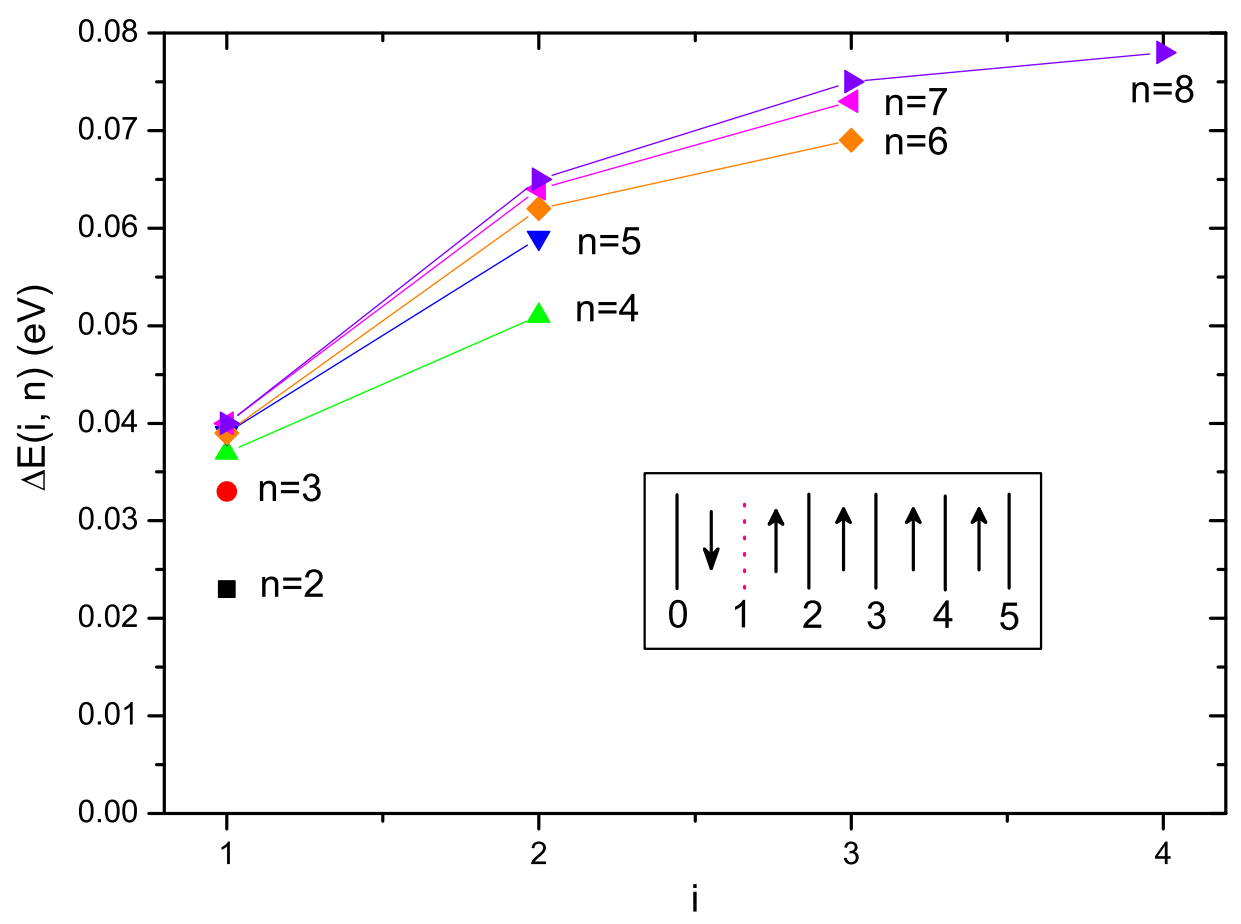

FIG. 7: (Color online) Site $i$ and cluster size $n$ dependence of the energy cost $\Delta E(i, n)$ to have an antiparallel magnetic moment pair. The site $i$ corresponds to the location of the $\mathrm{Bz}$ sandwiched with antiparallel magnetic moments of $\mathrm{V}$ and the cluster size $n$ corresponds to $\mathrm{V}_{n} \mathrm{Bz}_{n+1} \cdot t=t^{\prime}$ is assumed. In the inset, the configuration is specified with $i=1$ and $n=5$.

in Fig. 6. Without losing generality, $\epsilon_{p}$ can be taken as 0.0 to be the energy reference and $t^{\prime}$ is first assumed to be equal to $t . t, \epsilon_{d}$ and $\Delta$ are adjusted using the occupied four levels in the FM state and the values for the parameters are $t=1.648 \mathrm{eV}, \epsilon_{d}=-0.650 \mathrm{eV}$ and $\Delta=0.916 \mathrm{eV}$, respectively. Then in order to clarify the effects of variation of $t^{\prime} / t$ in the relative stability between FM and AFM states, we assume the following distance dependence of the $p-d$ hybridization, $t, t^{\prime} \propto d^{-4}$ with $d$ the distance between $\mathrm{C}$ and $\mathrm{V}$ and readjust $t$ and $t^{\prime}$ keeping $\epsilon_{d}, \Delta$ and the total energy of the FM state unchanged. According to our structural optimization for $U=3.0 \mathrm{eV}$, the $\mathrm{V}-\mathrm{C}$ distances are $2.304 \AA$ for the middle Bz and $2.248 \AA$ for the edge Bz. The final values of $t$ and $t^{\prime}$ are $1.555 \mathrm{eV}$ and $1.716 \mathrm{eV}$, respectively. By using these parameters, we find that $\mathrm{FM} \mathrm{V}_{2} \mathrm{Bz}_{3}$ is lower than the AFM one by 0.018 $\mathrm{eV}$. The corresponding value for the case of $t=t^{\prime}$ is $0.023 \mathrm{eV}$. For the cyclic case, FM state is more stable by $0.245 \mathrm{eV}$. These results are qualitatively consistent with our firstprinciples calculations. If a small contribution from $d \sigma$ states to AFM coupling may be 
taken into account, agreement between the model and the first-principles calculation may become better.

The model analysis can be easily extended to larger clusters to study the size effect. Here we show some results for $n=3$ and 4 cases. In larger clusters there are several magnetic configurations. Table II and Table III summarize the energy cost needed to reverse the spin magnetic moment of $\mathrm{V}$ from the ground state for $n=3$ and 4 , respectively. In the left-end column of the tables, arrows indicate the direction of $\mathrm{V}$ magnetic moment, while the vertical line corresponds to Bz. Those Bz's sandwiched with antiparallel V magnetic moments are denoted by dotted vertical lines. First, we note that in the GGA $+U$ scheme, the ground state is FM for both $n=3$ and 4 being consistent with other GGA-BLYP 18 calculations,,$\frac{5}{}$ though one of the GGA-PBE calculations claims that the AFM state is the ground state for $n=3 \stackrel{7}{?}$ Second, the energy cost for reversing the magnetic moment of one of the edge V's increases with $n$. This trend is seen in both the GGA $+U$ calculation and the model analysis. Third, the energy cost is basically controlled by the number of dotted vertical lines , i.e., the number of antiparallel pairs, in the magnetic configuration. Fourth, between the two configurations with one dotted vertical line (the 2nd and 3rd configurations in Table III), the one with antiparallel spin configuration at the cluster edge costs less energy. The second and the fourth aspects are clearly seen in Fig. 7 where the results by model calculations are also shown for larger clusters.

\section{CONCLUSION}

By examining the electronic structures of the infinite chain of $\mathrm{VBz}$ obtained with the GGA $+U$ calculations, we first found that the orbitals with different symmetries play distinct roles in the physical properties of the VBz system. The $d \sigma$ orbital of $\mathrm{V}$, which has very weak hybridization with $p \sigma$ orbital of $\mathrm{Bz}$, is localized and singly occupied with a large exchange splitting. It contributes one $\mu_{B}$ magnetic moment to the whole system but plays minor role in the magnetic coupling between neighboring V local moments. Perhaps, it may contribute to weak anti-ferromagnetic superexchange. The $d \pi$ and $p \pi$ states hybridize strongly to push the $\pi$ molecular orbitals far away from the Fermi level either fully occupied or empty. Therefore,

although they contribute to the stability of the multiple-decker sandwich structure, they are inactive in magnetic or electronic properties. The hybridization between $d \delta$ and $p \delta$ states 
is medium among the three types of bonds and these states are crucially important in the magnetic coupling. We pointed out that the mechanism of ferromagnetism stability proposed by Kanamori and Terakura ${ }^{12}$ is directly applicable to the ferromagnetism of the infinite chain of $\mathrm{VBz}$. We have also found that the presence of edge $\mathrm{Bz}$ in $\mathrm{V}_{2} \mathrm{Bz}_{3}$ cluster introduces an interesting modification to the mechanism. The edge Bz's are not simply inactive in the magnetic coupling but tend to favor the antiferromagnetic coupling of $\mathrm{V}$ magnetic moments. We set up a simple tight-binding model to analyze the problem in detail. Finally we studied the size dependence of magnetic interaction in the $\mathrm{V}_{n} \mathrm{Bz}_{n+1}$ clusters.

\section{Acknowledgments}

We thank Prof. H. Fukuyama and Prof. A. Nakajima for valuable comments and discussion. This work is partly supported by the Next Generation Supercomputing Project, Nanoscience Program and also partly by Grant-in-Aids for Scientific Research in Priority Area "Anomalous Quantum Materials", both from MEXT, Japan.

* Author to whom correspondence should be addressed. E-mail:hmweng@jaist.ac.jp

1 N. J. Long, Metallocenes; Blackwell Science: Oxford, 1998.

2 K. Miyajima, A. Nakajima, S. Yabushita, M. B. Knickelbein, K. Kaya, J. Am. Chem. Soc. 126, $13202(2004)$.

3 K. Miyajima, S. Yabushita, M. B. Knickelbein, and A. Nakajima, J. Am. Chem. Soc. 129, 8473 (2007).

4 T. Yasuike and S. Yabushita, J. Phys. Chem. A 103, 4533 (1999).

5 J. Wang, P. H. Acioli, and J. Jellinek, J. Am. Chem. Soc. 127, 2812 (2005).

6 A. K. Kandalam, B. K. Rao, P. Jena, R. Pandey, J. Chem. Phys. 120, 10414 (2004).

7 Y. Mokrousov, N. Atodiresei, G. Bihlmayer, S. Blügel, Int. J. Quan. Chem. 106, 3208 (2006).

8 M. B. Knickelbein, J. Chem. Phys. 121, 5281 (2004).

9 M. M. Rahman, H. Kasai, and E. S. Dy, Jpn. J. Appl. Phys. 44, 7954 (2005).

10 V. V. Maslyuk, A. Bagrets, V. Meded, A. Arnold, F. Evers, M. Brandbyge, T. Bredow, and I. Mertig, Phys. Rev. Lett. 97, 097201 (2006). 
11 Hongjun Xiang, Jinlong Yang, J. G. Hou, and Qingshi Zhu, J. Am. Chem. Soc. 128, 2310 (2006).

12 J. Kanamori and K. Terakura, J. Phys. Soc. Jpn, 70, 1433 (2001).

13 http://www.openmx-square.org/

14 T. Ozaki, Phys. Rev. B 67, 155108 (2003); T. Ozaki and H. Kino, ibid. 69, 195113 (2004); J. Chem. Phys. 121, 10879 (2004); Phys. Rev. B 72, 045121 (2005).

15 J. P. Perdew, K. Burke, and M. Ernzerhof, Phys. Rev. Lett. 77, 3865 (1996).

16 J. M. Soler, E. Artacho, J. D. Gale, A. Garcia, J. Junquera, P. Ordejon, and D. Sanchez-Portal, J. Phys.: Condens. Matter 14, 2745 (2002) and references therein.

17 M. J. Han, T. Ozaki, and J. Yu, Phys. Rev. B 73, 045110 (2006).

18 A. D. Becke, Phys. Rev. A 38, 3098 (1988); C. Lee, W. Yang, R. G. Parr, Phys. Rev. B 37, $785(1988)$. 